\title{
Discovery of Dynamical 3-Space: Theory, Experiments and Observations-A Review
}

\author{
Reginald T. Cahill \\ School of Chemical and Physical Sciences, Flinders University, Adelaide 5001, Australia
}

Received 2013-10-19; Revised 2013-12-09; Accepted 2014-01-02

\begin{abstract}
The existence of space as a dynamical system was recently revealed in 2002 , following the discovery that the Michelson-Morley light speed anisotropy experiment of 1887 was not null. Numerous later experiments have confirmed that discovery, as reviewed here, with the latest being the discovery of a very simple and cheap nanotechnology quantum detector method for observing space and its fluctuations/turbulence: Gravitational waves. As well over the last ten years the dynamical theory for space has been under development and has now been successfully tested against experiment and astronomical observations, explaining, in particular, the observed characteristics of galactic black holes, galactic rotations and universe expansion, all without "dark matter" and "dark energy".
\end{abstract}

Keywords: Dynamical 3-Space, Gravitational Waves, Light Speed Anisotropy, Cosmology, Quantum Classical Transition

\section{INTRODUCTION}

One of the most significant experiments in the history of physics was the Michelson-Morley 1887 light speed anisotropy experiment. While they reported a non-null result, it has been accepted that the magnitude of the fringe shifts was too small to constitute a real effect of anisotropy. This famous "null" experiment resulted in the development of the spacetime union of space and time and to Special Relativity (SR). Subsequently Newtonian gravity was generalized to have a spacetime form. Along the way the idea that space might be a dynamical system was ignored. However Cahill and Kitto (2003) discovered that the calibration theory for the Michelson interferometer was incorrect, it actually required a Lorentzian treatment and it was then discovered that the instrument was nearly 2000 times less sensitive than assumed. That meant that the observed fringe shifts indicated an anisotropy of the speed of light as high as $\sim 500 \mathrm{~km} \mathrm{sec}^{-1}$. There have been numerous later experiments all confirming those results and using a variety of experimental techniques. The latest uses a nanotechnology zener diode quantum effect, Cahill (2013b), which not only has detected a space speed of $\sim 500 \mathrm{~km} \mathrm{sec}{ }^{-1}$, but does so by measuring the travel time for turbulence/gravitational waves in that space flow, relative to the earth and over large distances: Adelaide to London and Perth to London. Clearly these experimental results challenge the foundations of the spacetime paradigm and its assumption that the speed of light is isotropic. Even more challenging is the discovery, Cahill (2008; 2013a), that the SR formalism is exactly derivable from Galilean Relativity by a mere change of space and time coordinates. So SR is not about the existence or properties of space, but about a choice of a coordinate system wrt which the speed of light is isotropic by construction. The outcome of these developments is that it is a neo-Lorentz Relativity that is being confirmed by experiment and which has passed all experimental and observational tests. As well the experimental discovery of the dynamical space led to a natural and unique generalisation of Newtonian gravity. This new theory of gravity then explains many anomalies and does not need "dark matter" nor "dark energy". Here we review some of the key aspects of these developments.

Starting with Kepler's planetary orbit discoveries, Newton developed a theory of gravity that we now know to be flawed and which subsequently flawed the 
generalization by Hilbert and Einstein. After some 300 years there is now a futile search for "dark matter" and "dark energy". Newton's approach was to assume that Galileo's observations of matter having the same acceleration in free fall could be explained by assuming that the magnitude of a gravitational force acting on an object with inertial mass $\mathrm{m}$, was proportional to $\mathrm{m}$, in which case $\mathrm{m}$ also acted as a gravitational mass or charge. This entailed an equality of the inertial mass and the gravitational mass, which became known as the Weak Equivalence Principle. However, starting from Galileo's observations we can follow a different development and one based on the following: That the equal gravitational acceleration of objects with different masses was caused by the flow of space, which had that acceleration at the location of the masses and that lowmass matter acted as a probe of the space acceleration. This entails the idea that space exists, is dynamical and directly detectable. The derivation of the reaction of matter to the accelerating space had to await the development of the quantum theoryof matter and we find then that gravity is a refraction of the quantum waves and is thus an emergent phenomenon. We also briefly show that this account of gravity resolves the above anomalies and leads to new experimental phenomena and tests. We also discover that the dynamics of space has three parameters: (i) $G$ describing the dissipative flow of space into matter and which, for the case of the earth, has been directly detected by means of spacecraft earth-flyby Doppler shift data and (ii) $\alpha \approx 1 / 137$ - the fine structure constant, which determines a self-interaction coupling constant of the dynamical space and which bore hole $g$ and black hole mass data reveals to be the fine structure constant and (iii) $\delta$-a Planck-like distance, which is responsible for the absence of singularities, Cahill and Kerrigan (2011) and Rothall and Cahill (2013). So the new theory of space and gravity not only provides a well tested theory, but also points to a new unification of space, gravity and the quantum theory. It was pointed out in Cahill (2005) that this unification appears to arise from an informationtheoretic approach to comprehending reality, leading to a quantumfoam description of space. The new theory also explains various so-called relativistic effects, but in a way that does not involve "spacetime". Indeed the putative predictions of the "spacetime" formalism are falsified by experiments. Experiments confirm instead Lorentz's account of relativistic effects, as being caused by the absolute motion of objects wrt space and for which the maximum speed is c. Experiments show that the speed of light, in vacuum, is anisotropic for an observer moving through space, as 1st detected by Michelson and Morley in 1887 and that the flowing space affects both quantum matter and electromagnetic waves, via its time dependence and/or its velocity inhomogeneity. The dynamical space also exhibits wave/turbulence effects, usually called "gravitational waves" and again 1 st detected in this experiment. We emphasise that the dynamical space is not a hydrodynamical theory, with some entity flowing through a non-dynamical geometrical space.

The speed and direction of gravitational waves have been directly measured via waveform time delays from detectors located in Adelaide and London and separately from Perth and London, Cahill (2013b). The Adelaide to London correlations were detected utilising the discovery that so-called "clock jitter" in two Digital Storage Oscilloscopes (DSO) is actually correlated, with the London signal delayed relativeto the Adelaide signal by 13 to $20 \mathrm{sec}$, depending on sidereal time, so that at least part of the clock jitter is actually induced by passing gravitational waves. Subsequently similar correlations were discovered in Random Event Generator (REG) correlated data. These detect the quantum to classical transition for electrons tunnelling through a barrier in a tunnel diode, a nanotechnology device. According to the standard interpretation of quantum theory such electron current fluctuations should be completely random, which is why such devices are also known as hardware Random Number Generators (RNG) and have a variety of applications assuming such randomness.

These discoveries make the detection and study of gravitational waves particularly simple and easily extend to a network of detectors and for the Random Event Generators (REG) which employ zener diodes. This means that the REG network forms an international gravitational wave detector network and has existed since 1998 and so that data is an extremely valuable to the characterisation of the gravitational wave effect and also other phenomena which appear to be induced by more extreme fluctuations. Correlations of the gravitational wave forms permit determination of the speed and direction of space, which agrees with results from NASA Earth-flyby Doppler shift data and withthe 1925/26 Dayton Miller Mt.Wilson gas-mode Michelson interferometer data. The correlation data also reveals two new phenomena: A speed-up when the waves pass deeper into the earth and a wave reverberation effect, Cahill and Deane (2013). For collocated zener diodes the current fluctuations are highly correlated, with no time delay effects, as expected. The quantum to classical transition is thus 
shown to be caused by 3-space dynamics and so challenges the standard interpretation of probabilities in quantum theory.

\section{CLASSICAL PHYSICS GRAVITATIONAL WAVE DETECTORS}

Classical gravitational wave detectors have employed a number of physical effects and designs: Gas-mode Michelson interferometers, optical fibre Michelson interferometers, RF coaxial cable travel time differential measurements and more compact RF coaxial cableoptical fibre measurements, spacecraft Earth-flyby Doppler effects and dual RF coaxial cable travel time measurements Cahill (2009b; 2012). All of these techniques utilise light or EMR anisotropy speed effect in a single device. The key issue with such devices is that they are single-site devices and require a calibration theory, which depends upon an assumed theory. For example the sensitivity of a Michelson interferometer, as indicated by the travel time difference between the two arms and detected by means of fringe shifts as the detector is rotated, is given by:

$\Delta \mathrm{t}=\mathrm{k}^{2} \frac{\mathrm{Lu}_{\mathrm{p}}^{2}}{\mathrm{c}^{3}} \cos (2(\theta-\psi)$

where, $\mathrm{L}$ is the arm length, $\mathrm{u}_{\mathrm{P}}$ is the speed projected onto the plane of the interferometer and the angles measure the rotation, Cahill (2009b). Equation (1) is applied to the data in conjunction with terms accounting for the inclined mirrors and temperature drift effects. The critical factor $\mathrm{k}^{2}$ is the calibration constant. With a gas present in the light path, with refractive index $n, k^{2} \approx n^{2}-$ 1 to a good approximation. Results from two gas-mode Michelson interferometer experiments are shown in Fig. 1 and 2. The results reveal significant turbulence, which has been identified as gravitational waves and much greater in magnitude than expected. Michelson and Morley in the 1 st such experiment in 1887 assumed that $\mathrm{k}^{2}=1$, whereas with air present, $\mathrm{n}=1.00029$, giving $\mathrm{k}^{2} \approx$ 0.0006 and so much less sensitive than assumed. Note that a vacuum-mode Michelson interferometer has $\mathrm{k}^{2}=0$ and so completely insensitive to gravitational waves.

A recent gravitational wave experiment used differential travel time measurements in a dual RF coaxial cable array, Cahill (2012). This technique relies upon the absence of Fresnel drag in RF coaxial cables, at least for low RFfrequencies $(\sim 10 \mathrm{MHz})$. The results agree with those from the Miller gas-mode Michelson interferometer and from the NASA flyby Doppler shift data (Fig. 3). The fluctuations were again observed to be a $20 \%$ effect.

The interpretation of the magnitude of the detected effects in these classical detector experiments all rely upon some calibration theory and there has always been confusion. Fortunately spacecraft flyby Doppler shift analysis does not suffer from such problems and has indeed confirmed the results from the classical detectors. We now report the discovery that nanotechnology quantum detectors respond to the fluctuations of the passing space and when the data from two well-separated detectors is subject to a correlation analysis of the two local waveforms the average speed and direction of the passing space is revealed, together with significant wave/turbulence effects. This technique gives an absolute measurement of travel times.

\section{QUANTUM GRAVITATIONAL WAVE DETECTORS}

When extending the Dual RF Coaxial Cable Detector experiment to include one located in London, in addition to that located in Adelaide, an analysis of the measured Digital Storage Oscilloscope (DSO) internal noise in each identically setup instrument was undertaken, when the extensive RF coaxial cable array was replaced by short leads. This was intended to determine the $\mathrm{S} / \mathrm{N}$ ratio for the joint Adelaide-London experiment. Surprisingly the internal noise was found to be correlated, with the noise in the London DSO being some 13 to 20 seconds behind the Adelaide DSO noise, Cahill (2013b). LeCroy WaveRunner 6051ADSOs were used. The correlation data had a phase that tracked sidereal time, meaning that the average direction was approximately fixed wrt the galaxy, but with extensive fluctuations as well from the gravitational wave/turbulence effect, that had been seen in all previous experiments. The explanation for this DSO effect was not possible as the DSO is a complex instruments and which component was responding to the passing space fluctuations could not be determined. But the correlation analysis did demonstrate that not all of the internal noise in the DSO was being caused solely by some random process intrinsic to the instrument. Subsequent experiments, below, now suggest that there are zener diodes within the time difference measurements hardware within the DSO.

The travel time delay $\tau(\mathrm{t})$ was determined by computing the correlation function:

$$
C(\tau, t)=\int_{t-T}^{t+T} d t^{\prime} S_{1}\left(t^{\prime}-\tau / 2\right) S_{2}\left[t^{\prime}+\tau / 2\right) e^{-\alpha\left(t^{\prime}-t\right)^{2}}
$$



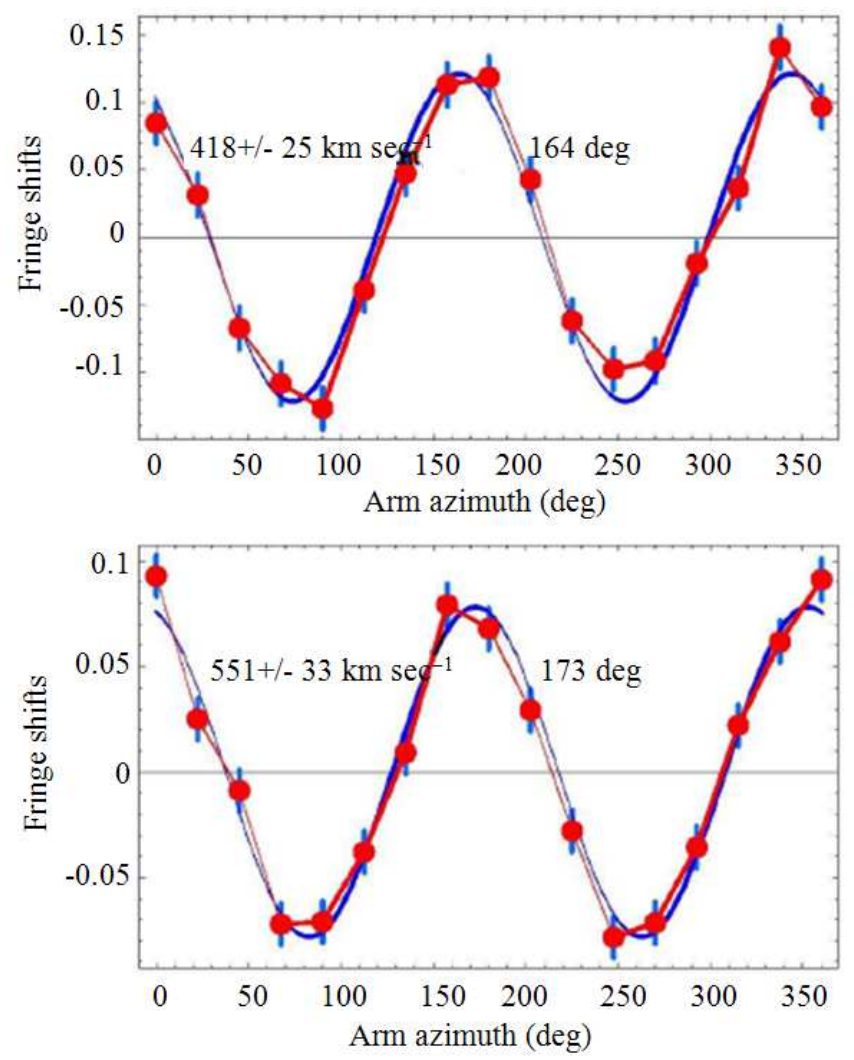

Fig. 1. A typical Miller averaged-data from September 16, 1925, $4^{\mathrm{h}} 40^{\prime}$ ' Local Sidereal Time (LST) -an average of data from 20 turns of the gas-mode Michelson interferometer. Plot and data after fitting and then subtracting both the temperature drift and Hicks effects from both, leaving the expected sinusoidal form (b) Best result from the Michelson-Morley 1887 data-an average of 6 turns, at $7^{\mathrm{h}}$ LST on July 11, 1887. In both cases the indicated speed is $\mathrm{v}_{\mathrm{p}}$-the 3 -space speed projected onto the plane of the interferometer. The angle is the azimuth of the 3 -space speed projection at the particular LST. The speed fluctuations from day to day significantly exceed these errors and reveal the existence of 3-space flow turbulence-i.e., gravitational waves

for the two detector signals $\mathrm{S}_{1}(\mathrm{t})$ and $\mathrm{S}_{2}(\mathrm{t})$. Here $2 \mathrm{~T}=$ $200 \mathrm{~s}$ is the time interval used, about UTC time $t$. The Gaussian term ensures the absence of end-effects. Maximising $C(\tau, \mathrm{t})$ wrt $\tau$ gives $\tau(\mathrm{t})$-the delay time vs UTC $t$ and plotted in Fig. 4 and 5, where the data has been binned into $1 \mathrm{~h}$ time intervals and the rms also shown. The speed and direction, over a $24 \mathrm{~h}$ period, was determined by fitting the time delay data using:

$$
\tau=\frac{\mathrm{R} \cdot \mathrm{v}}{\mathrm{v}^{2}}
$$

where, $\mathrm{R}$ is the Adelaide-London spatial separation vector and $\mathrm{v}(\theta, \delta)$ is the 3 -space velocity vector, parametrised bya speed, RA and Declination. This expression assumes a plane wave form for the gravitational waves. The $\tau(\mathrm{t})$ delay times show large fluctuations, corresponding to fluctuations in speed and/or direction, as also seen in data in Fig. 1.
There are much simpler devices that were discovered to also display time delayed correlations over large distances: These are the Random Number Generators (RNG) or Random Event Generators (REG). There are various designs available from manufacturers and all claim that these devices manifest hardware random quantum processes, as they involve the quantum to classical transition when a measurements, say, of the quantum tunnelling of electrons through a nanotechnology potential barrier, $\sim 10 \mathrm{~nm}$ thickness, is measured by a classical/macroscopic system. According to the standard interpretation of the quantum theory, the collapse of the electron wave function to one side or the other of the barrier, after the tunnelling produces a component on each side, is purely a random event, internal to the quantum system. However this interpretation had never been tested experimentally. 


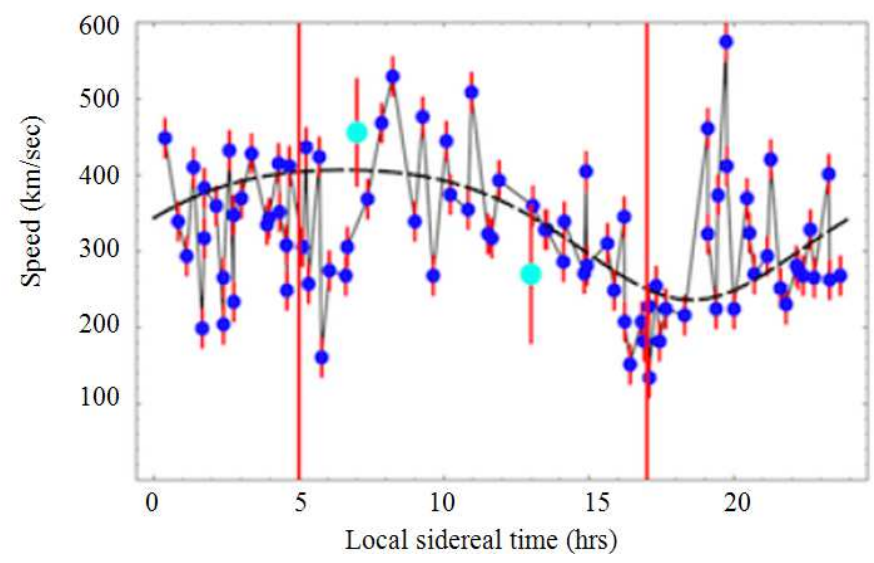

Fig. 2. Speeds $v_{p}$, of the space velocity $v$ projected onto the horizontal plane of the Miller gas-mode Michelson interferometer located atop Mt.Wilson, plotted against local sidereal, for a composite day, with data collected over a number of days in September 1925, Cahill (2009b). The data shows considerable fluctuations, from hour to hour and also day to day, as this is a composite day. The dashed curve shows the non-fluctuating best-fit variation over one day, as the earth rotates, causing the projection onto the plane of the interferometer of the velocity of the average direction of the space flow to change. The maximum projected speed from the curve is $417 \mathrm{~km} \mathrm{sec}^{-1}$, corresponding to a speed of $453 \mathrm{~km} \mathrm{sec}^{-1}$, with a RA of $\sim 5 \mathrm{~h}$, which is very close to results reported herein. The Cassini flyby Doppler shift data in August 1999 gives a RA $=5.2 \mathrm{~h}$, Cahill (2009b). The green data points, with error bars, at $7 \mathrm{~h}$ and $13 \mathrm{~h}$, are from the Michelson-Morley 1887 data. The $\sim 20 \%$ speed fluctuations are seen to be much larger than statistically determined errors, revealing the presence of turbulence in the space flow, i.e., gravitational waves

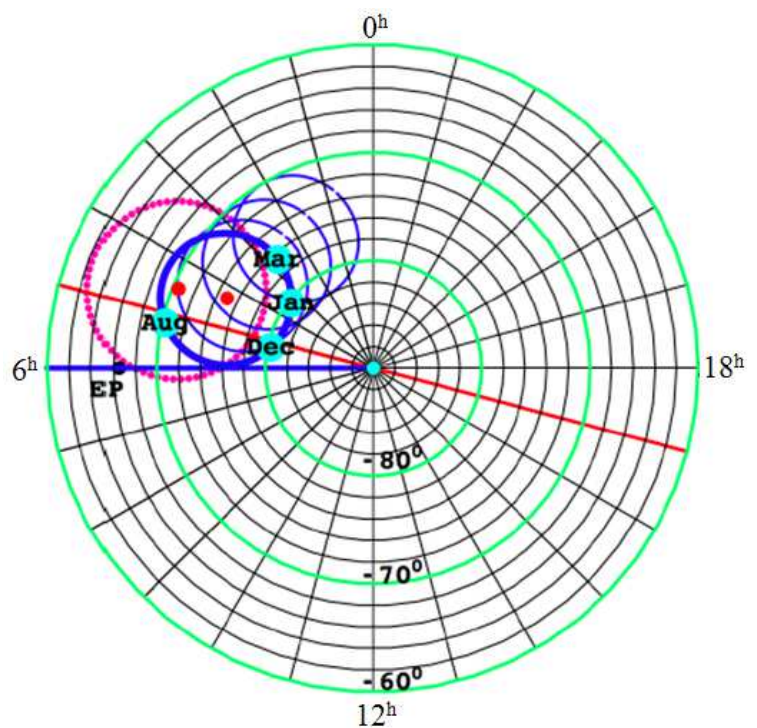

Fig. 3. South celestial pole region. The dot (red) at RA $=4.3^{\mathrm{h}}, \mathrm{Dec}=75^{\circ} \mathrm{S}$ and with speed $486 \mathrm{~km} \mathrm{sec}^{-1}$, is the direction of motion of the solar system through space determined from NASA spacecraft earth-flyby Doppler shifts, Cahill (2009b), revealing the EM radiation speed anisotropy. The thick (blue) circle centred on this direction is the observed velocity direction for di_erent days of the year, caused by earth orbital motion and sun 3-space inflow. The corresponding results from the Miller gas-mode interferometer are shown by 2 nd dot (red) and its aberration circle (red dots). For December 8, 1992, the velocity is RA = $5.2^{\mathrm{h}}$, Dec $=80^{\circ} \mathrm{S}$, speed $491 \mathrm{~km} \mathrm{sec}^{-1}$, see table of Cahill $(2009 \mathrm{~b})$. The thinner blue aberration circles relate to determination of earth 3 -space inflow speed 


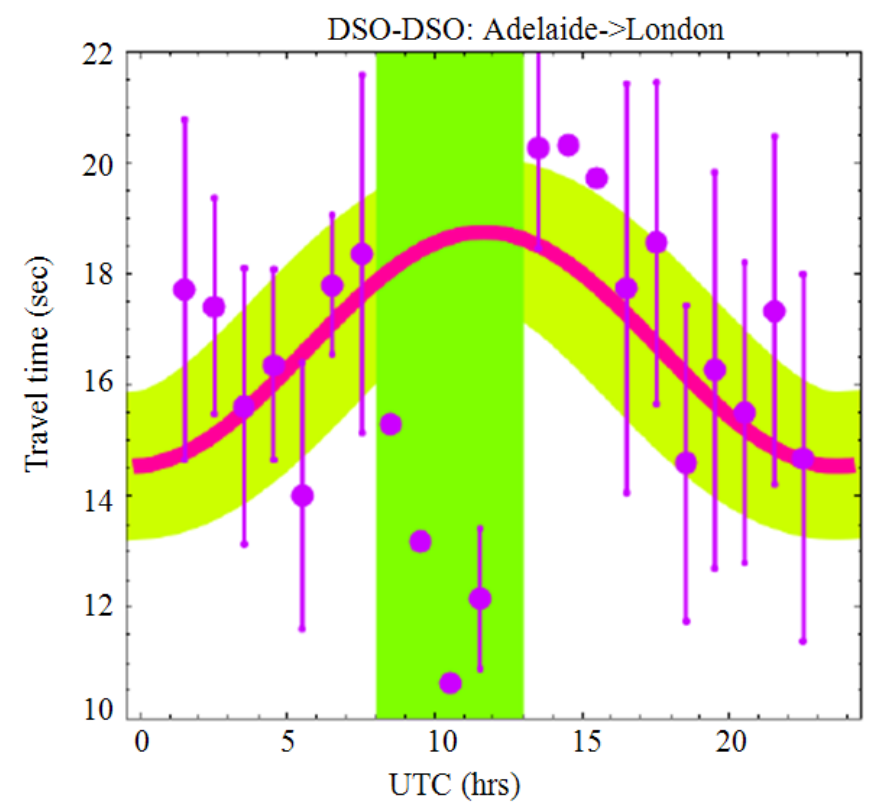

Fig. 4. Travel times from DSO-DSO Adelaide-London data, January 1, 2013. The data in each $1 \mathrm{~h}$ interval has been binned and the average and rms shown. The thick (red line) shows best fit to data using plane wave travel time predictor, but after excluding those data points between 8 and $13 \mathrm{~h} \mathrm{UTC} \mathrm{(top)} \mathrm{indicated} \mathrm{by} \mathrm{vertical} \mathrm{band.} \mathrm{Those} \mathrm{data} \mathrm{points} \mathrm{are} \mathrm{not} \mathrm{consistent} \mathrm{with} \mathrm{the} \mathrm{plane} \mathrm{wave} \mathrm{modelling}$ and suggest a scattering process when the waves pass deeper into the earth. The Perth-London phase is retarded wrt AdelaideLondon phase by $\sim 1.5 \mathrm{~h}$, consistent with Perth being $1.5 \mathrm{~h}$ west of Adelaide. The Adelaide-London data gives speed $=512 \mathrm{~km} \mathrm{sec}^{-1}$, $\mathrm{RA}=4.8 \mathrm{~h}, \mathrm{Dec}=83^{\circ} \mathrm{S}$ and the Perth-London data gives speed $=528 \mathrm{~km} \mathrm{sec}^{-1}, \mathrm{RA}=5.3 \mathrm{~h}, \mathrm{Dec}=81^{\circ} \mathrm{S}$. The broad band tracking the best fit line is for $+/-1 \mathrm{sec}$ fluctuations, corresponding to speed fluctuation of $+/-17 \mathrm{~km} \mathrm{sec}^{-1}$

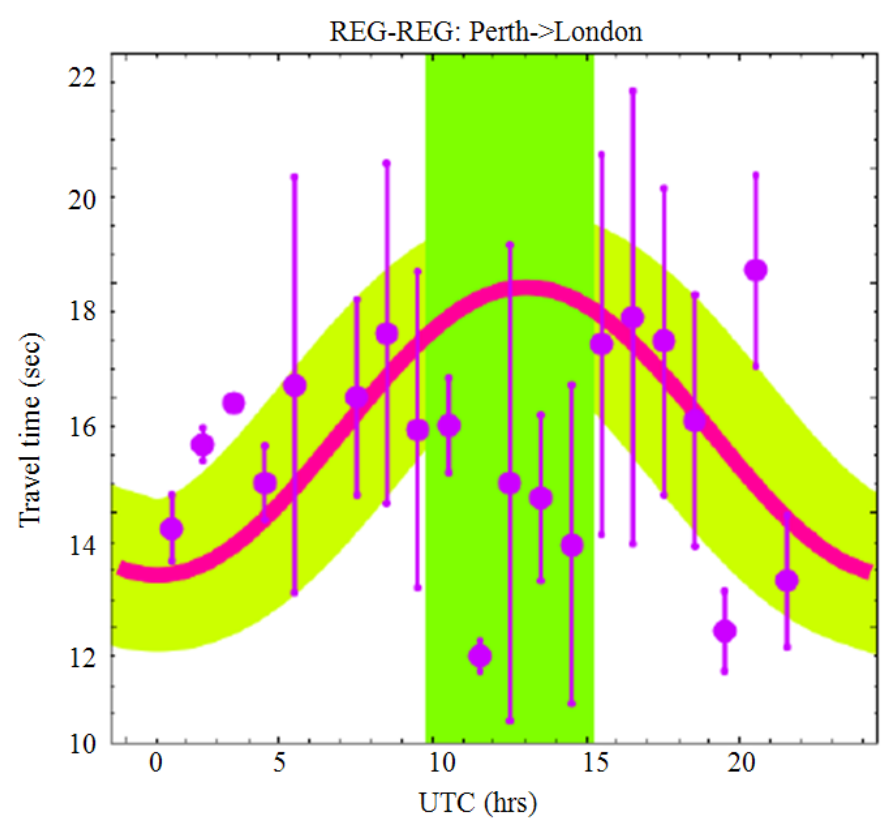

Fig. 5. Travel times from REG-REG Perth-London data, January 1, 2013, giving speed $=528 \mathrm{~km} \mathrm{sec}^{-1}, \mathrm{RA}=5.3 \mathrm{~h}$, Dec $=81^{\circ} \mathrm{S}$ 
Guided by the results from the DSO correlated-noise effect, the data from two REGs, located in Perth and London, was examined. The data, from the GCP international network: http://teilhard.global-mind.org, showed the same correlation effect as observed in the DSO experiments.

\section{DYNAMICAL THEORY OF SPACE}

We begin the heuristic derivation of the dynamics of space and the emergence of gravity as a quantum matter effect, by assuming that Galileo's observations suggest the existence of a dynamical space, whose acceleration will be shown to determine the same acceleration of matter and whose time dependence and inhomogeneity of velocity determines the observed anisotropy of the speed of light and causing light bending and gravity as refraction effects. Physics must employ a covariance formulation, in the sense that ultimately predictions are independent of observers and that there must also be a relativity principle that relates observations by different observers. We assume then that space has a structure whose movement, wrt an observer, is described by a velocity field, $v(r, t)$, at the classical physics level, at a location $r$ and time $t$, as defined by the observer. In particular the space coordinates $r$ define an embedding space, which herein we take to be Euclidean. At a deeper level space is probably a fractal quantum foam, which is only approximately embeddable in a 3-dimensional space at a coarse-grained level, Cahill (2005; 2009a; 2011a). This embedding space has no ontological existence-it is not real. Ironically Newton took this space to be real but unobservable and so a different concept and so excluding the possibility that gravity was caused by an accelerating space. It is assumed that different observers, in relative uniform motion, relate their description of the velocity field by means of the Galilean Relativity Transformation for positions and velocities. It is usually argued that the Galilean Relativity Transformations were made redundant and in error by the Special Relativity Transformations. However this is not so there exist an exact linear mapping between Galilean Relativity and Special Relativity (SR), differing only by definitions of space and time coordinates Cahill (2008). This implies that the so-called Special Relativity (SR) relativistic effects are not actual dynamical effectsthey are purely artifacts of a peculiar choice of space and time coordinates. In particular Lorentz symmetry is merely a consequence of this choice of space and time coordinates and is equivalent to Galilean symmetry. Nevertheless Lorentz symmetry remains valid, even though a local preferred frame of reference exists. Lorentz Relativity, however, goes beyond Galilean Relativity in that the limiting speed of systems wrt to the local space causes various so-called relativist effects, such as length contractions and clock dilations.

The Euler covariant constituent acceleration a $(r, t)$ of space is then defined by:

$$
\begin{gathered}
a=\lim _{\Delta t \rightarrow 0} \frac{v(r+v(r, t) \Delta t, t+\Delta t)-v(r, t)}{\Delta t} \\
=\frac{\partial v}{\partial t}+(v . \nabla) v
\end{gathered}
$$

which describes the acceleration of a constituent element of space by tracking its change in velocity. This means that space has a (quantum) structure that permits its velocity to be defined and detected, which experimentally has been done. We assume here that the flow has zero vorticity $\nabla \times \mathrm{v}=0$ and then the flow is determined by a scalar function $\mathrm{v}=\nabla_{\mathrm{u}}$. We then need one scalar equation to determine the space dynamics, which we construct by forming the divergence of a. The inhomogeneous term then determines a dissipative flow caused by matter, expressed as a matter density and where the coefficient turns out to be Newton's gravitational constant:

$$
\nabla \cdot\left(\frac{\partial \mathrm{v}}{\partial \mathrm{t}}+(\mathrm{v} . \nabla) \mathrm{v}\right)=4 \pi \mathrm{G} \rho(\mathrm{r}, \mathrm{t})
$$

Note that even a time independent matter density or even the absence of matter can be associated with a timedependent flow. This equation follows essentially from covariance and dimensional analysis. For a spherically symmetric matter distribution, of total mass $M$ and a time-independent spherically symmetric flow we obtain from the above and external to the sphere of matter, the acceleration of space:

$$
\mathrm{v}(\mathrm{r})=-\sqrt{\frac{2 \mathrm{GM}}{\mathrm{r}}} \hat{\mathrm{r}} \text {, giving } \mathrm{a}(\mathrm{r})=-\frac{\mathrm{GM}}{\mathrm{r}^{2}} \hat{\mathrm{r}}
$$

which is the inverse square law. Newton applied such an acceleration to matter, not space and which Newton invented directly by examining Kepler's planetary motion laws, but which makes no mention of what is 
causing the acceleration of matter, although in a letter in 1675 to Oldenburg, Secretary of the Royal Society and later to Robert Boyle, he speculated that an undetectable ether flow through space may be responsible for gravity. Here, however, the inverse square law emerges from the Euler constituent acceleration, which imposes a space self-interaction. At the surface of the earth the in-flow speed is $11 \mathrm{~km} \mathrm{~s}^{-1}$ and the sun in-flow speed at $1 \mathrm{AU}$ is $42 \mathrm{~km} \mathrm{~s}^{-1}$, with both detected Cahill (2009b). If the sphere of matter is in motion, asymptotically wrt space, then the flow equation becomes non-trivial to solve and no analytic solutions are known. Numerical solutions reveal nontrivial wave effects. Note that one cannot go from a flow of space associated with, say matter asymptotically stationary wrt to space, to the case where the matter is moving, asymptotically, wrt to space-these are very differentdynamical situations. But in either case it is trivial to transform the velocity field, using Galilean Relativity, between different observers who are in relative motion.

While the above 3-space dynamical equation followed from covariance and dimensional analysis, this derivation is not complete yet. One can add additional terms with the same order in speed and spatial derivatives and which cannot be a priori neglected. There are two such terms, as in:

$$
\begin{aligned}
\nabla \cdot\left(\frac{\partial \mathrm{v}}{\partial \mathrm{t}}+(\mathrm{v} \cdot \nabla) \mathrm{v}\right) & +\frac{5 \alpha}{4}\left((\operatorname{trD})^{2}-\operatorname{tr}\left(\mathrm{D}^{2}\right)\right)+\ldots \\
& =-4 \pi \mathrm{G} \rho
\end{aligned}
$$

where, $D_{i j}=\partial v_{i} / \partial x_{j}$. However to preserve the inverse square law external to a sphere of matter, when the matter is stationary, asymptotically, wrt space, the two terms must have coefficients $\alpha$ and $-\alpha$, as shown. Here is a dimensionless space self-interaction coupling constant. The ellipsis denotes higher order derivative terms with dimensioned coupling constants, which come into play when the flow speed changes rapidly wrt separation. However the observed dynamics of stars and gas clouds near the centre of the Milky Way galaxy has revealed the need for such a term and we find that the space dynamics there requires an extra term:

$$
\begin{aligned}
& \nabla \cdot\left(\frac{\partial \mathrm{v}}{\partial \mathrm{t}}+(\mathrm{v} \cdot \nabla) \mathrm{v}\right)+\frac{5 \alpha}{4}\left((\operatorname{trD})^{2}-\operatorname{tr}\left(\mathrm{D}^{2}\right)\right)+ \\
& +\delta^{2} \nabla^{2}\left((\operatorname{trD})^{2}-\operatorname{tr}\left(\mathrm{D}^{2}\right)\right)+\ldots=-4 \pi \mathrm{G} \rho
\end{aligned}
$$

where, $\delta$ has the dimensions of length and appears to be a very small Planck-like length, Cahill and Kerrigan (2011).

This then gives us the dynamical theory of 3-space. It can be thought of as arising via a derivative expansion from a deeper theory, such as a quantum foam theory, Cahill (2005). Note that the equation does not involve $\mathrm{c}$, is nonlinear and time-dependent and involves non-local direct interactions. Its success implies that the universe is more connected than previously thought. Even in the absence of matter there can be time-dependent flows of space. To test this theory we need to determine how quantum matter and EM radiation respond to this dynamical space. We note immediately that this dynamics is very rich in that various new phenomena emerge and which have been observed and which do not occur in Newtonian gravity, which is a linear theory, nor in its relativistic generalisation, General Relativity (GR), with both being one-parameter theories, G: Essentially GR is flawed by the assumption that GR must reduceto Newtonian gravity in the non-relativistic low-mass limit.

\section{QUANTUM MATTER AND EMERGENT GRAVITY}

We now derive, uniquely, how quantum matter responds to the dynamical 3 -space. This gives the 1 st derivation of the phenomenon of gravity and reveals this to be a quantum matter wave refraction effect. For a freefall quantum system with massmthe Schrodinger equation is uniquely generalized Cahill (2006), with the new terms required to maintain that the motion is intrinsically wrt the 3 -space and not wrt the embedding space and that the time evolution is unitary:

$$
\mathrm{i} \hbar \frac{\partial \psi(\mathrm{r}, \mathrm{t})}{\partial \mathrm{t}}=-\frac{\hbar^{2}}{2 \mathrm{~m}} \nabla^{2} \psi(\mathrm{r}, \mathrm{t})-\mathrm{i} \hbar\left(\mathrm{v} . \nabla+\frac{1}{2} \nabla . \mathrm{v}\right) \psi(\mathrm{r}, \mathrm{t})
$$

The space and time coordinates $\{t, x, y, z\}$ ensure that the separation of a deeper and unified process into different classes of phenomena-here a dynamical 3-space (quantum foam) and a quantum matter system, is properly tracked and connected. As well the same coordinates may be used by an observer to also track the different phenomena. A quantum wave packet propagation analysis gives the matter acceleration $\mathrm{g}=$ $\mathrm{d}^{2}<\mathrm{r}>/ \mathrm{dt}^{2}$ induced by wave refraction to be: 


$$
\begin{aligned}
& \mathrm{g}=\frac{\partial \mathrm{v}}{\partial \mathrm{t}}+(\mathrm{v} \cdot \nabla) \mathrm{v}+(\nabla \times \mathrm{v}) \times \mathrm{v}_{\mathrm{R}} \\
& +\mathrm{v}_{\mathrm{R}}\left(\mathrm{r}_{0}(\mathrm{t}), \mathrm{t}\right)=\mathrm{v}_{0}(\mathrm{t})-\mathrm{v}\left(\mathrm{r}_{0}(\mathrm{t}), \mathrm{t}\right)
\end{aligned}
$$

where, $v_{R}$ is the velocity of the wave packet relative to the 3-space and where $v_{O}$ and $r_{O}$ are the velocity and position relative to the observer. The last term generates the Lense-Thirring effect as a vorticity driven effect. In the limit of zero vorticity we obtain that the quantum matter acceleration is the same as the 3-space acceleration: $\mathrm{g}=\mathrm{a}$. This confirms that the new physics is in agreement with Galileo's observations that all matter falls with the same acceleration. Using arcane language this amounts to a derivation of the weak equivalence principle.

Significantly the quantum matter 3-space-induced 'gravitational' acceleration also follows from maximising the elapsed proper time wrt the quantum matter wave-packet trajectory $\mathrm{r}_{\mathrm{o}}(\mathrm{t})$, Cahill (2005):

$$
\tau=\int d t \sqrt{1-\frac{v_{R}^{2}\left(r_{0}(t), t\right)}{c^{2}}}
$$

which entails that matter has a maximum speed of $\mathrm{c}$ wrt to space and not wrt an observer. This maximisation ensures that quantum waves propagating along neighbouring paths are in phase-the condition for a classical trajectory. This gives:

$$
\begin{aligned}
\mathrm{g}= & \frac{\partial \mathrm{v}}{\partial \mathrm{t}}+(\mathrm{v} \cdot \nabla) \mathrm{v}+(\nabla \times \mathrm{v}) \times \mathrm{v}_{\mathrm{R}} \\
& -\frac{\mathrm{v}_{\mathrm{R}}}{1-\mathrm{v}_{\frac{\mathrm{R}}{2}}^{2}} \frac{1}{2} \frac{\mathrm{d}}{\mathrm{dt}}\left(\frac{\mathrm{v}_{\mathrm{R}}^{2}}{\mathrm{c}^{2}}\right)+\ldots
\end{aligned}
$$

and then taking the limit $\mathrm{v}_{\mathrm{R}} / \mathrm{c} \rightarrow 0$ we recover the nonrelativistic limit, above. This shows that (i) the matter 'gravitational' geodesic is a quantum wave refraction effect, with the trajectory determined by a Fermat maximum propertime principle and (ii) that quantum systems undergo a local time dilation effect. The last, relativistic, term generates the planetary precession effect. If clocks are forced to travel different trajectories then the above predicts different evolved times when they again meet-this is the Twin Effect, which now has a simple and explicit physical explanation-it is an absolute motion effect, meaning motion wrt space itself. This elapsed proper time expression invokes Lorentzian relativity, that the maximum speed is c wrt to space and not wrt the observer, as in Einstein SR. The differential proper time has the form:

$$
c^{2} d \tau^{2}=c^{2} d t^{2}-(d r-v(r, t) d t)^{2}=g_{\mu \nu} d x^{\mu} d x^{v}
$$

Which defines an induced metric for a curved spacetime manifold. However this has no ontological significance and the metric is not determined by GR.

\section{ELECTROMAGNETIC RADIATION AND DYNAMICAL SPACE}

We must generalise the Maxwell equations so that the electric and magnetic fields are excitations within the dynamical 3-space and not of the embedding space. The minimal form in the absence of charges and currents is:

$$
\begin{aligned}
& \nabla \times \mathrm{E}=-\mu_{0}\left(\frac{\partial \mathrm{H}}{\partial \mathrm{t}}+\mathrm{v} . \nabla \mathrm{H}\right), \nabla \cdot \mathrm{E}=0 \\
& \nabla \times \mathrm{H}=\epsilon_{0}\left(\frac{\partial \mathrm{E}}{\partial \mathrm{t}}+\mathrm{v} . \nabla \mathrm{E}\right), \nabla \cdot \mathrm{H}=0
\end{aligned}
$$

which was first suggested by Hertz (1890), but with v then being only a constant vector field and not interpreted as a moving space effect. As easily determined the speed of EM radiation is now $\mathrm{c}=1 / \sqrt{\mu_{0} \varepsilon_{0}}$ with respect to the 3 -space and not wrt an observer in motion through the 3-space. The Michelson-Morley 1887 experiment 1st detected this anisotropy effect, as have numerous subsequent experiments. A time-dependent and/or inhomogeneous velocity field causes the refraction of EM radiation. This can be computed by using the Fermat least-time approximation-the opposite of that for quantum matter. This ensures that EM waves along neighbouring paths are in phase. Then an EM ray path $\mathrm{r}(\mathrm{t})$ is determined by minimising the elapsed travel time:

$$
T=\int_{s_{i}}^{s_{f}} \frac{d s\left|\frac{d r}{d s}\right|}{\left|c \hat{v}_{R}(s)+v(r(s), t(s))\right|}
$$

with $v_{R}=\frac{d r}{d t}-v(r(t), t)$ by varying both $r(s)$ and $t(s)$, finally giving $r(t)$. Here $s$ is an arbitrary path parameter 
and $\hat{c} v_{R}$ is the velocity of the EM radiation wrt the local 3 -space, namely c. The denominator is the speed of the $=$ EM radiation wrt the observer's Euclidean spatial coordinates. This equation may also be used to calculate the gravitational lensing by black holes, filaments, Cahill (2011b) and by ordinary matter, using the appropriate 3space velocity field. It produces the measured light bending by the sun. In particular galactic lensing agrees with observational data and does not require "dark matter".

\section{EARTH BORE HOLES DETERMINE $\alpha$}

The value of the parameter was first determined from earth bore hole g-anomaly data, which shows that gravity decreases more slowly down a bore hole than predicted by Newtonian gravity. Using the new theory of gravity we find the borehole gravity anomaly at radius $\mathrm{r}=\mathrm{R}+\mathrm{d}$ to be, with $d<0$, is, Cahill (2006):

$$
\Delta \mathrm{g}=\mathrm{g}_{\mathrm{NG}}(\mathrm{d})-\mathrm{g}(\mathrm{d})=20 \pi \alpha \mathrm{G} \rho(\mathrm{R}) \mathrm{d}+\mathrm{O}\left(\alpha^{2}\right)
$$

The experimental data then reveals $\alpha$ to be the fine structure constant, to within experimental errors, Fig. 6.

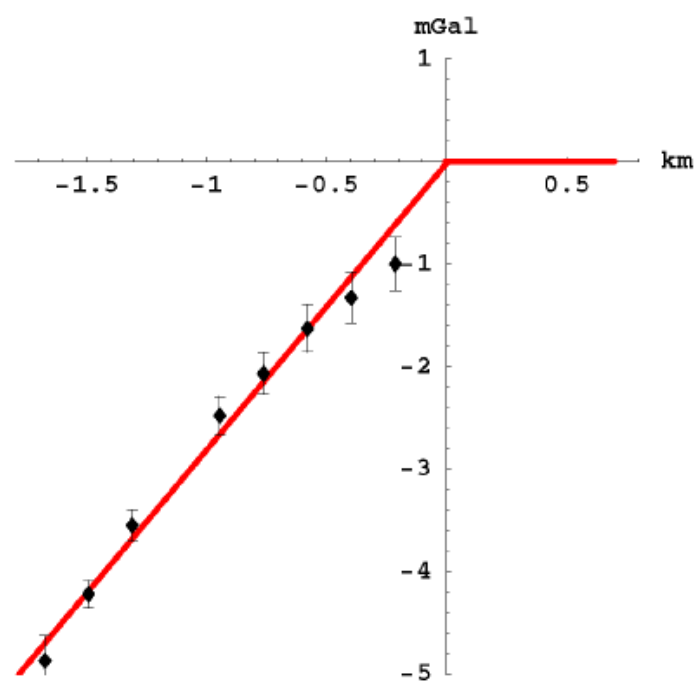

Fig. 6. Small the data shows the gravity residuals for the Greenland Ice Shelf Airy measurements of the g(r) profile, defined as $\Delta \mathrm{g}(\mathrm{r})=\mathrm{g}_{\text {Newton }}-\mathrm{g}_{\text {observed }}$ and measured in $\mathrm{mGal}\left(1 \mathrm{mGal}=10^{-3} \mathrm{~cm} / \mathrm{s}^{2}\right)$ and plotted against depth in $\mathrm{km}$. The borehole effect is that Newtonian gravity and the new theory differ only beneath the surface. We obtain $\alpha^{-1}=137.9 \pm 5$ from fitting the slope of the data

\section{G MEASUREMENT ANOMALIES}

There has been a long history of anomalies in the laboratory measurements of Newton's gravitational constant G. The explanation is that the gravitational acceleration external to a piece of matter is only given by application of Newton's inverse square law for the case of an isolated spherically symmetric mass and using an external small test mass. Forother shapes and with finite size test masses, the $\alpha$-dependent interaction results in forces that differ from Newtonian gravity at $\mathrm{O}(\alpha)$, as observed. This implies that laboratory measurements to determine $\mathrm{G}$ will also measure $\alpha$.

\section{DISPENSING WITH DARK MATTER}

Combining the 3-space zero-vorticity dynamics with the quantum matter acceleration, we obtain:

$$
\nabla . \mathrm{g}=-4 \pi \mathrm{G} \rho-4 \pi \mathrm{G} \rho_{\mathrm{DM}}, \nabla \times \mathrm{g}=0
$$

where we define:

$$
\rho_{\mathrm{DM}}=\frac{5 \alpha+4 \delta \nabla^{2}}{16 \pi \mathrm{G}}\left((\operatorname{trD})^{2}-\operatorname{tr}\left(\mathrm{D}^{2}\right)\right)
$$

This is Newtonian gravity but with the extra dynamical term. The role of this expression is to reveal that if we analyse gravitational phenomena we will usually find that the matter density $\rho$ is insufficient to account for the observed g. Until recently this failure of Newtonian gravity has been explained away as being caused by some unknown and undetected but real "dark matter" density. This expression shows that to the contrary it is a dynamical property of 3-space itself. In deference to that language we call $\rho_{\mathrm{DM}}$ the 3space induced effective dark matter density. From observed galactic EM lensing and galactic star trajectories $\rho_{\mathrm{DM}}$ may be determined and compared with the dynamical 3-space dynamics.

\section{EXPANDING UNIVERSE: DISPENSING WITH DARK ENERGY}

The dynamical 3-space theory has a time dependent expanding universe solution of the Hubble form. In the 
absence of matter, $v(r, t)=H(t) r$ with $H(t)=1 /(1+\alpha / 2) t$, giving a scale factor $a(t)=\left(t / t_{0}\right)^{4 /(4+\alpha)}$, predicting essentially a uniform expansion rate. This gives a parameter free account of the supernovae magnitude-redshift data, Cahill (2009a) and Cahill and Rothall (2012). That data reveals a uniformly expanding universe. However the Friedmann equations from GR do not have such a uniformly expanding solution and ad hoc "dark matter" and "dark energy" terms are added to "save the theory", giving the current standard cosmological model. Best fitting the $\Omega_{\Lambda}$ and $\Omega_{\mathrm{DM}} \Lambda \mathrm{CDM}$ composition parameters to the above solution gives $\Omega_{\Lambda}=$ 0.73 and $\Omega_{\mathrm{DM}}=0.27$, the same values as determined by fitting the $\Lambda \mathrm{CDM}$ to the supernova data (Fig. 9). This demonstrates that "dark matter" and "dark energy" are epicycles of GR. Extending that model into the future leads to the spurious claim that the universe will undergo an exponential rate of expansion.

\section{BLACK HOLES}

In the absence of matter the dynamical 3-space equation has black hole solutions of the asymptotic form $\mathrm{v}(\mathrm{r})^{2} \approx \mathrm{A} \frac{\delta}{\mathrm{r}}+\mathrm{B}\left(\frac{\delta}{\mathrm{r}}\right)^{5 \alpha}$ when $\mathrm{r}>>\delta$, giving $\mathrm{g}(\mathrm{r})=\mathrm{GM}(\mathrm{r}) / \mathrm{r}^{2}$ (Fig. 7).
Where:

$$
M(r)=M_{0}+M_{0}\left(\frac{r}{r_{s}}\right)^{1-5 \alpha}
$$

This is precisely the form observed for the black hole at the centre of the Milky Way, Fig. 10, Cahill and Kerrigan (2011) and Rothall and Cahill (2013). For $r>r_{s}$ these black holes produce a $1 / \mathrm{r}$ gravitational acceleration and not $\mathrm{a} 1 / \mathrm{r}^{2}$ form as assumed in the usual Newtoniangravity based analysis. This then produces flat rotation curves, as shown in Fig. 8. NG and GR required the invention of dark matter to "explain" this effect.

\section{COSMIC FILAMENTS}

The 3-space dynamics also has cosmic filament solutions, asymptotically, $v(r)=-\mu / r^{5 \alpha / 4}$, where $r$ is here the perpendicular distance from the filament, for arbitrary $\mu$. The gravitational acceleration is long-range and attractive to matter, i.e., $g$ is directed inwards towards the filament, $g(r)$ $=-\alpha \mu^{2} / 8 r^{1+5 / 2}$. This is for a single infinite-length filament. It is conjectured that more complex solutions involving a network of filaments and black holes exist and which explain the observed cosmic web (Fig. 11).

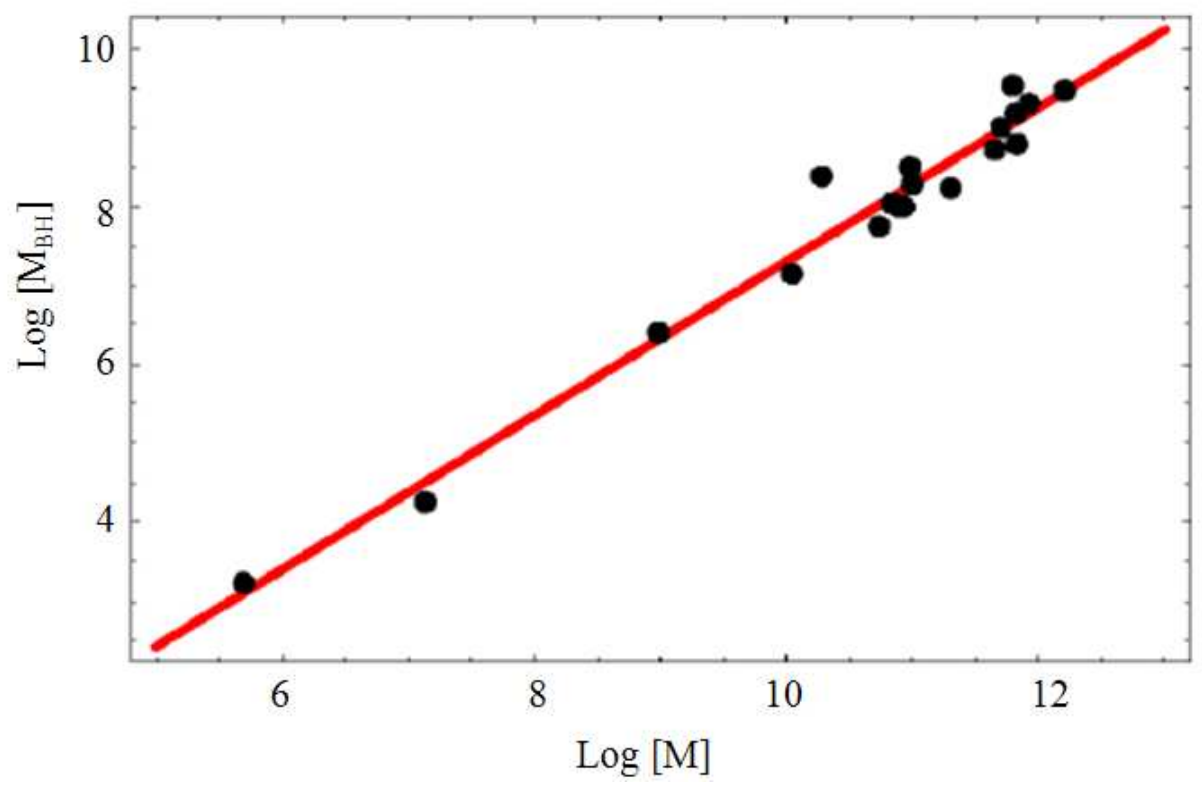

Fig. 7. The data shows black hole masses $\mathrm{M}_{\mathrm{BH}}$ for a variety of spherical matter systems, from Milky Way globular clusters to spherical galaxies, with masses $M$, plotted against $\log _{10}[M]$, in solar masses $M_{0}$. The straight line is $M_{B H}=\frac{\alpha}{2} M$ 
Reginald T. Cahill / American Journal of Space Science 1 (2): 77-93, 2013

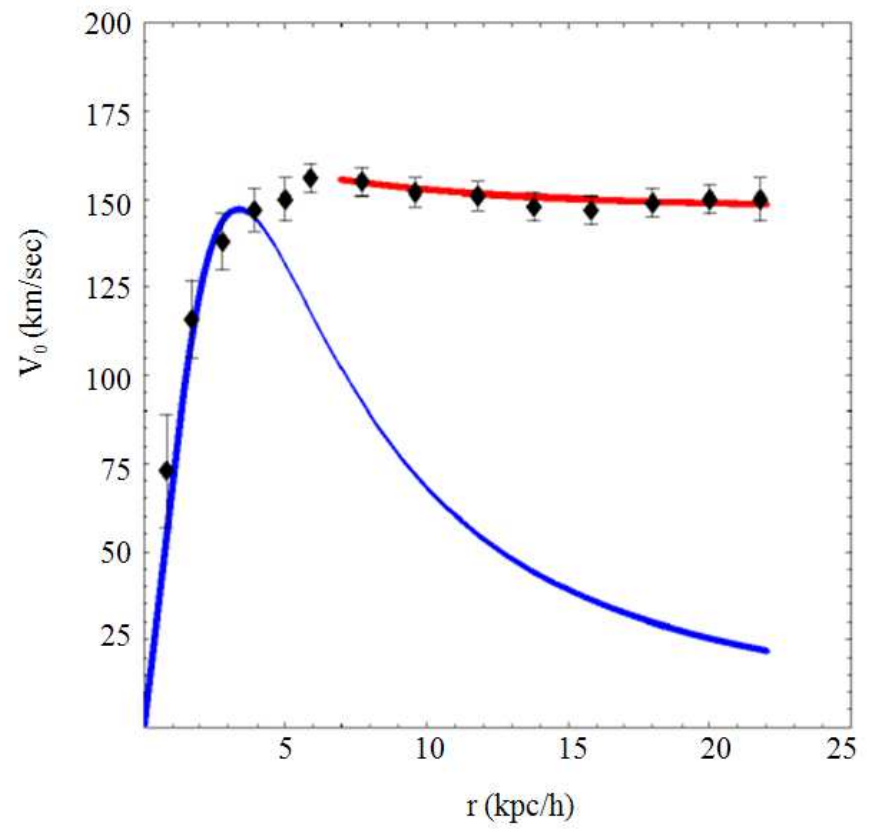

Fig. 8. Plots of the rotation speed data for the spiral galaxy NGC3198. Lower curve shows Newtonian gravity prediction, while upper curve shows asymptotic flat rotation speeds

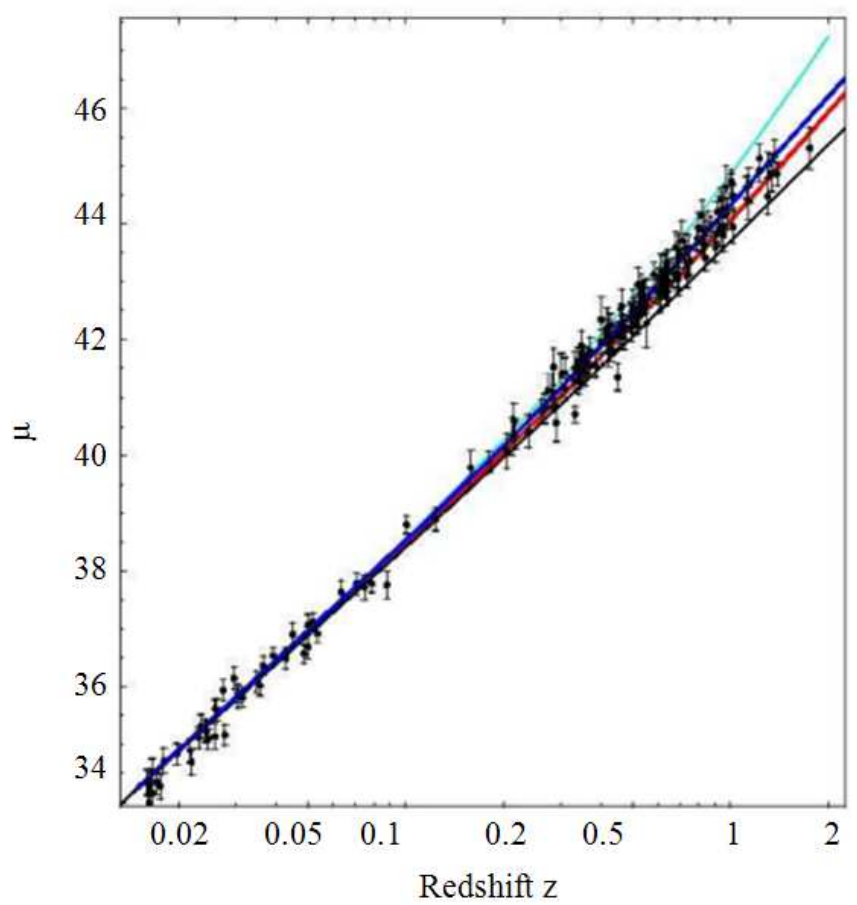

Fig. 9. Supernovae magnitude-redshift data. Upper curve (light blue) is "dark energy" only $\Omega_{\Lambda}=1$. Next curve (blue) is best fit of "dark energy"-"dark-matter" $\Omega_{\Lambda}=0.73$. Lowest curve (black) is "dark matter" only $\Omega_{\Lambda}=0.2$ nd lowest curve (red) is generic uniformly expanding universe 


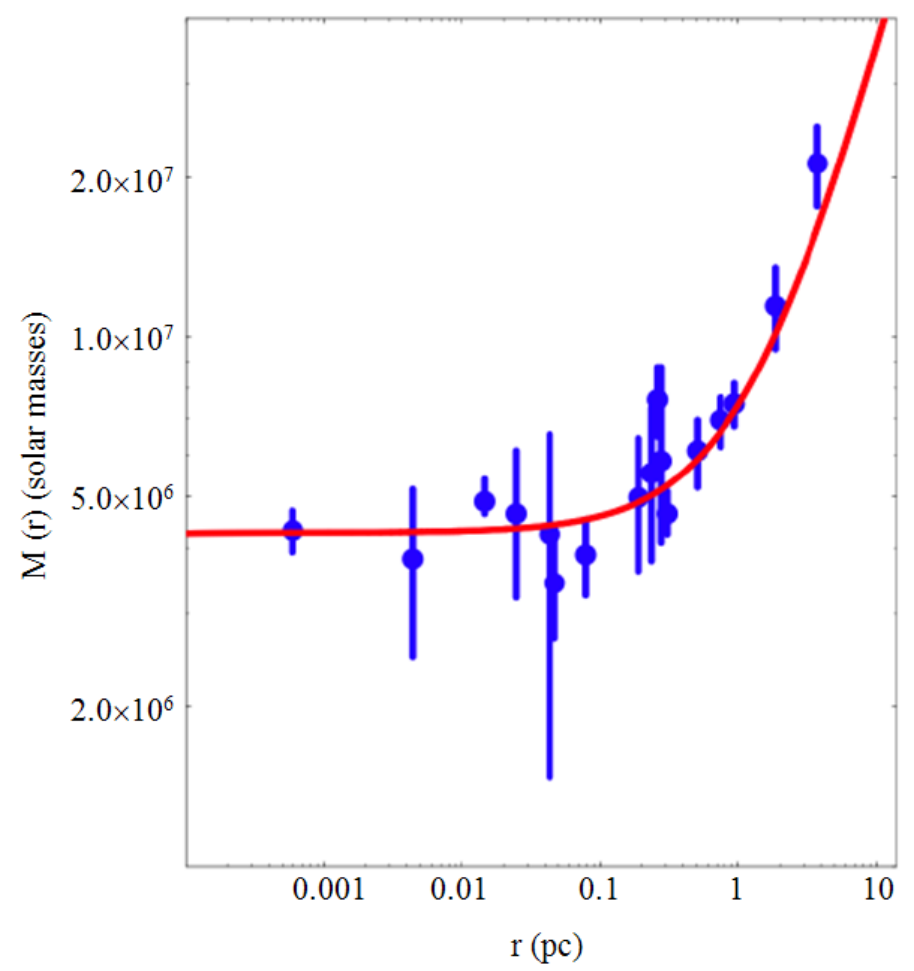

Fig. 10. The $M(r)$ data for the Milky Way SgrA* black hole, showing the flat regime, that mimics a point-like mass and the rising form beyond $r_{s}=1.33 p c$. Here $M_{0}$ and $r_{s}$ parametrise a quantum foam soliton and involves no actual matter. The data gives $\mathrm{M}_{0}=4.5 \pm 0.4 \times 10^{6}$ solar masses

\section{SPECIAL RELATIVITY FROM GALILEAN RELATIVIT}

The assumptions in GaR are (i) space exists, but is not observable and not dynamical and is modelled as a Euclidean 3-space $\left(\mathrm{E}^{3}\right)$, which entails the notion that space is without structure, (ii) observers measure space and time intervals using rods and clocks, whose respective lengths and time intervals are not affected by their motion through space, (iii) velocities are measured relative to observers, where different observers, $\mathrm{O}$ and O', relate their space and time coordinates by:

$$
t^{\prime}=t, x^{\prime}=x-V t, y^{\prime}=y, z^{\prime}=z
$$

where, $\mathrm{V}$ is the relative speed of the observers (in their common $\mathrm{x}$-direction, for simplicity). The speed $\mathrm{w}$ of an object or waveform (in the $\mathrm{x}$ direction) according to each observer, is related by:

$$
\mathrm{w}^{\prime}=\mathrm{w}-\mathrm{V}
$$

Equation (2) and (3) form the Galilean Relativity Transformation and the underlying assumptions define Galilean Relativity (GaR). Newton based his dynamics on Galilean Relativity, in particular his theory of gravity, to which General Relativity reduces in the limit of low mass densities and low speeds.

When Maxwell formulated his unification of electric and magnetic fields, the speed of EM waves came out to be the constant $\mathrm{c}-1 / \sqrt{\varepsilon_{0} \mu_{0}}$ for any observer and so independent of the motion of the observers wrt one another or to space. This overtly contradicted GaR, in (3). Hertz (1890) pointed out the obvious fix-up, namely that Maxwell had mistakenly not used the then-known Euler constituent derivative $\partial / \partial \mathrm{t}+\mathrm{v} . \nabla$, in place of $\partial / \partial \mathrm{t}$, where $\mathrm{v}$ is the velocity of some structure to space relative to an observer, in which case Maxwell's equations would only be valid in the local rest frame defined by this structure. In that era a dual model was then considered, namely with a Euclidean space $\mathrm{E}^{3}$ and an extended allfilling aether substance, so that the velocity $\mathrm{v}$ was the velocity of the aether relative to an observer. 


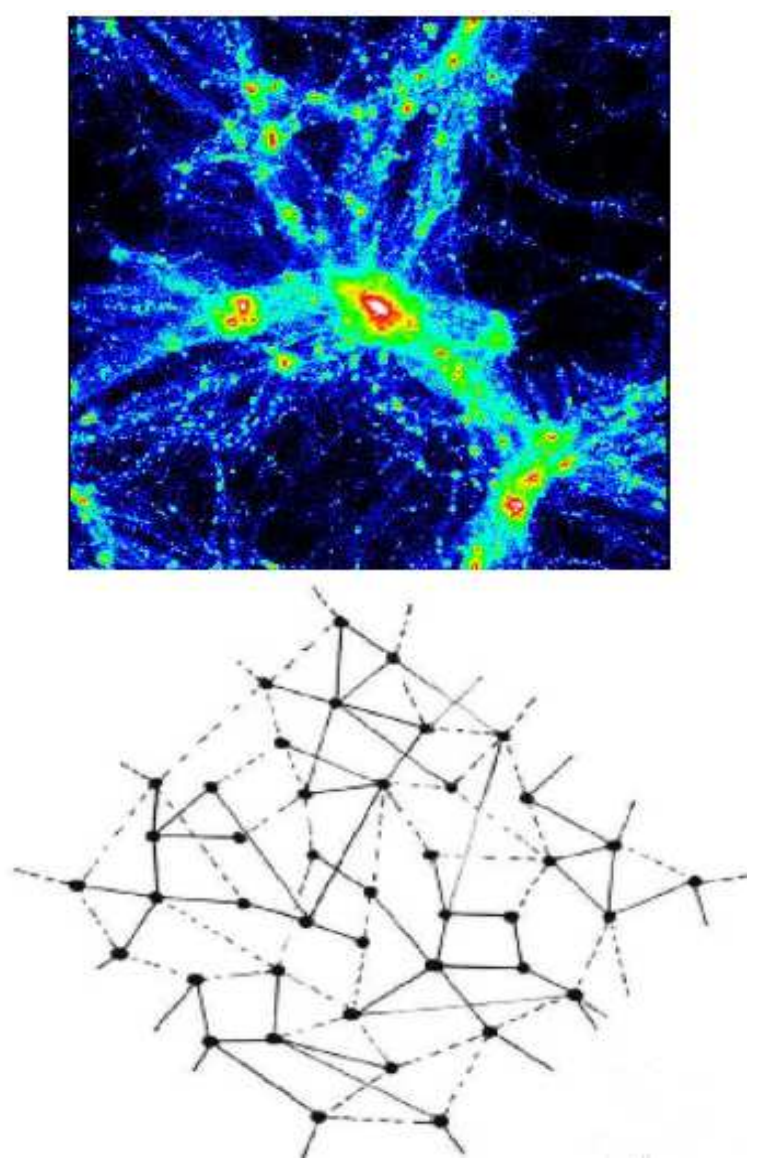

Fig. 11. Top: Cosmic filaments as revealed by gravitational lensing statistical tomography. From J.A. Tyson and G. Bernstein, Bell Laboratories, Physical Sciences Research,

http://www.belllabs.com/org/physicalsciences/project s/darkmatter/darkmatter.html. Bottom: Cosmic network of primordial filaments and primordial black holes.

To be explicit let us consider the case of electromagnetic waves, as described by the vector potential A(r, t) satisfying the wave equation (in absence of charges and currents), but using the Euler constituent derivative, as suggested by Hertz Equation (4):

$$
\left(\frac{\partial}{\partial \mathrm{t}}+\mathrm{v}(\mathrm{r}, \mathrm{t}) \cdot \nabla\right)^{2} \mathrm{~A}(\mathrm{r}, \mathrm{t})=\mathrm{c}^{2} \nabla^{2} \mathrm{~A}(\mathrm{r}, \mathrm{t})
$$

Here $\nabla=\left\{\frac{\partial}{\partial x}, \frac{\partial}{\partial y}, \frac{\partial}{\partial z}\right\}$. In Lorentz Relativity there is a static aether in addition to an actual Euclidean space, so $\mathrm{v}$ is independent of $\mathrm{r}$ and $\mathrm{t}$; whereas in neo-Lorentz Relativity $v(r, t)$ describes a dynamical space, with $r$ and $\mathrm{t}$ describing a cosmic embedding space and a cosmic time. We find plane-wave solutions only for the case where the space flow velocity, relative to an observer, is locally time and space independent, viz uniform:

$$
\mathrm{A}(\mathrm{r}, \mathrm{t})=\mathrm{A}_{0} \sin (\mathrm{k} \cdot \mathrm{r}-\omega \mathrm{t})
$$

with $\omega(\mathrm{k}, \mathrm{v})=\mathrm{c}|\overrightarrow{\mathrm{k}}|+\mathrm{v} \cdot \mathrm{k}$. The EM wave group velocity is then:

$$
\mathrm{v}_{\mathrm{g}}=\vec{\nabla}_{\mathrm{k}} \omega(\mathrm{k}, \mathrm{v})=\mathrm{ck}+\mathrm{v}
$$

and we see that the wave has velocity $\mathrm{v}_{\mathrm{g}}$ relative to the observer, with the space flowing at velocity $\mathrm{v}$ also relative to the observer and so the EM speed is c in direction $\hat{k}$ relative to the aether (LR) or space (nLR). In searching for experimental evidence for the existence of this aether, or more generally a Preferred Frame of Reference (PFR), Michelson conceived of his interferometer. Unknown to Michelson was that his design had an intrinsic fatal flaw: If operated in vacuum mode it was incapable of detecting the PFR effect, while with air present, as operated by Michelson and Morley in 1887, it was extremely insensitive, Cahill and Kitto (2003). The problem was that Michelson had used Newtonian physics, viz GaR, in calibrating the interferometerMichelson and Morley detected fringe shifts, but they were smaller than expected and were interpreted as a null effect: There was no aether or PFR effect. However Lorentz and Fitzgerald offered an alternative explanation: Physical objects, such as the arms supporting the interferometer optical elements, undergo a contraction in the direction of movement through the aether, or more generally relative to the PFR: The length becoming $L=L_{0} \sqrt{1-v_{R}^{2} / c^{2}}$, where $L_{0}$ is the physical length when at rest wrt the PFR and $v_{R}$ is the speed relative to the PFR. It must be noted that this is not the Lorentz contraction effect predicted by SR, as discussed later, as that involves $\mathrm{L}=\mathrm{L}_{0} \sqrt{1-\mathrm{v}_{0}^{2} / \mathrm{c}^{2}}$, where $\mathrm{v}_{\mathrm{O}}$ is the speed of the arm or any space interval relative to the observer. The difference between these two predictions is stark and has been observed experimentally and the SR prediction is proven wrong, Cahill (2013a).

Next consider two observers, $\mathrm{O}$ and $\mathrm{O}^{\prime}$, in relative motion. Then the actual intrinsic or physical time and space coordinates of each are, in both LR and nLR, related by the Galilean transformation and here we 
consider only a uniform v: These coordinates are not the directly measured distances/time intervals-they require corrections to give the intrinsic values. We have taken the simplest case where $\mathrm{V}$ is the intrinsic relative speed of the two observers in their common $\mathrm{x}$ directions. Then from (2) the derivatives are related by:

$$
\frac{\partial}{\partial \mathrm{t}}=\frac{\partial}{\partial \mathrm{t}^{\prime}}-\mathrm{V} \frac{\partial}{\partial \mathrm{x}^{\prime}}, \frac{\partial}{\partial \mathrm{x}}=\frac{\partial}{\partial \mathrm{x}^{\prime}} \frac{\partial}{\partial \mathrm{y}}=\frac{\partial}{\partial \mathrm{y}^{\prime}} \frac{\partial}{\partial \mathrm{z}}=\frac{\partial}{\partial \mathrm{z}^{\prime}}
$$

In the general case space rotations may be made. Then (4) becomes for the 2nd observer, with v' $=\mathrm{v}-\mathrm{V}$ Equation (5):

$$
\left(\frac{\partial}{\partial \mathrm{t}}+\mathrm{v}(\mathrm{r}, \mathrm{t}) \cdot \nabla\right)^{2} \mathrm{~A}\left(\mathrm{r}, \mathrm{t}^{\prime}\right)=\mathrm{c}^{2} \nabla^{\prime 2} \mathrm{~A}^{\prime}\left(\mathrm{r}^{\prime}, \mathrm{t}^{\prime}\right)
$$

with $A^{\prime}\left(r^{\prime}, t^{\prime}\right)=A(r, t)$. If the flow velocity $v(r, t)$ is not uniform then we obtain refraction effects for the EM waves, capable of producing gravitational lensing. Only for an observer at rest in a time independent and uniform aether (LR) or space (nLR) does v' disappear from (5).

The above uses physically intrinsic choices for the time and space coordinates, which are experimentally accessible. However we could choose to use a new class of time and space coordinates, indicated by upper-case symbols T, X, $\mathrm{Y}, \mathrm{Z}$, that mixes the above time and space coordinates. We begin by showing that Special Relativity (SR), with its putative spacetime as the foundation of reality, is nothing more than Galilean Relativity (GaR) written in terms of these mixed space and time coordinates. The failure to discover this, until 2008, Cahill (2008), reveals one of the most fundamental blunders in physics. One class of such mixed coordinatesfor $\mathrm{O}$ is Equation (6):

$$
\mathrm{T}=\gamma(\mathrm{v})\left(\left(1-\frac{\mathrm{v}^{2}}{\mathrm{c}^{2}}\right) \mathrm{t}+\frac{\mathrm{vx}}{\mathrm{c}^{2}}\right)
$$

$\mathrm{X}=\gamma(\mathrm{v}) \mathrm{x}, \mathrm{Y}=\mathrm{y}, \mathrm{Z}=\mathrm{z}$

where, $\mathrm{v}$ is the uniform speed of space (in the $\mathrm{x}$ direction) and where $\gamma(\mathrm{v})=1 / \sqrt{1-\mathrm{v}^{2} / \mathrm{c}^{2}}$. Note that this is not a Lorentz transformation. If an object has speed $\mathrm{w}$, $\mathrm{x}=\mathrm{wt}$, wrt to $\mathrm{O}$, then it has speed $\mathrm{W}, \mathrm{X}=\mathrm{WT}$, using the mixedcoordinates, wrt $\mathrm{O}$ Equation (7):

$$
\mathrm{W}=\frac{\mathrm{w}}{1-\frac{\mathrm{v}^{2}}{\mathrm{c}^{2}}+\frac{\mathrm{v}}{\mathrm{c}^{2}} \mathrm{~W}}
$$

Similarly for O' using v', w' and W'. In particular (7) gives for the relative speed of $\mathrm{O}^{\prime}$ wrt $\mathrm{O}$ in the mixed coordinates Equation (8):

$$
\overline{\mathrm{V}}=\frac{\mathrm{V}}{1-\frac{\mathrm{v}^{2}}{\mathrm{c}^{2}}+\frac{\mathrm{v}}{\mathrm{c}^{2}} \mathrm{~V}}
$$

Using the above we may now express the Galilean speed transformation in terms of $\mathrm{W}, \mathrm{W}$ and $\overline{\mathrm{V}}$ for the mixed coordinates, giving Equation (9):

$$
\mathrm{W}^{\prime}=\frac{\mathrm{W}-\overline{\mathrm{V}}}{1-\mathrm{W} \overline{\mathrm{V}} / \mathrm{c}^{2}}
$$

which is the usual SR transformation for speeds, but here derived exactly from the Galilean transformation. Note that $\mathrm{c}$ enters here purely because of the definitions in (6), which is designed to ensure that wrt the mixed spacetime coordinates the speed of light is invariant: c. To see this note that from (6) the transformations for the derivatives are found to be Equation (10):

$$
\begin{aligned}
& \frac{\partial}{\partial \mathrm{t}}=\gamma(\mathrm{v})\left(1-\frac{\mathrm{v}^{2}}{\mathrm{c}^{2}}\right) \frac{\partial}{\partial \mathrm{T}}, \\
& \frac{\partial}{\partial \mathrm{x}}=\gamma(\mathrm{v})\left(\frac{\mathrm{v}}{\mathrm{c}^{2}} \frac{\partial}{\partial \mathrm{T}}+\frac{\partial}{\partial \mathrm{X}}\right), \\
& \frac{\partial}{\partial \mathrm{y}}=\frac{\partial}{\partial \mathrm{y}}, \frac{\partial}{\partial \mathrm{z}}=\frac{\partial}{\partial \mathrm{Z}} \\
& \bar{\nabla}=\left\{\frac{\partial}{\partial \mathrm{X}}, \frac{\partial}{\partial \mathrm{Y}}, \frac{\partial}{\partial \mathrm{Z}}\right\} . \text { Then we have from }(4), \text { for uniform: } \\
& \qquad\left(\frac{\partial}{\partial \mathrm{T}}\right)^{2} \overline{\mathrm{A}}(\mathrm{R}, \mathrm{T})=\mathrm{c}^{2} \bar{\nabla}^{2} \overline{\mathrm{A}}(\mathrm{R}, \mathrm{T})
\end{aligned}
$$

with $\mathrm{R}=\{\mathrm{X}, \mathrm{Y}, \mathrm{Z}\}$ and $\overline{\mathrm{A}}(\mathrm{R}, \mathrm{T})=\mathrm{A}(\mathrm{r}, \mathrm{t})$. The speed of EM waves is now c for all observers. This is a remarkable result. In the new class of coordinates the dynamical equation no longer contains the space velocity $\mathrm{v}$-it has been mapped out of the dynamics. The EM dynamics is now invariant under Lorentz transformations Equation (11):

$$
\begin{aligned}
& \mathrm{T}^{\prime}=\gamma(\overline{\mathrm{V}})\left(\mathrm{T}-\frac{\overline{\mathrm{V}} \mathrm{X}}{\mathrm{c}^{2}}\right), \\
& \mathrm{X}^{\prime}=\gamma(\overline{\mathrm{V}})(\mathrm{X}-\overline{\mathrm{V}} \mathrm{T}), \mathrm{Y}^{\prime}=\mathrm{Y}, \mathrm{Z}^{\prime}=\mathrm{Z}
\end{aligned}
$$

and we note that for two events with coordinate differences $\{\mathrm{dT}, \mathrm{dX}\}$ or $\left\{\mathrm{dT}^{\prime}, \mathrm{dX}^{\prime}\right\}$ Equation (12): 
$\mathrm{dl}^{2} \equiv \mathrm{c}^{2} \mathrm{dT}^{\prime 2}-\mathrm{dX} \mathrm{X}^{2}=\mathrm{c}^{2} \mathrm{dT}^{2}-\mathrm{dX^{2 }}$

defines the invariant interval for different observers.

There is now no reference to the underlying flowing space: For an observer using this class of space and time coordinates the speed of EM waves relative to the observer is always $\mathrm{c}$ and so invariant-there will be no EM speed anisotropy. We could also introduce, following Minkowski, "spacetime" light cones along which $\mathrm{d}^{2}=\mathrm{dT}^{2}-\mathrm{dR}^{2} / \mathrm{c}^{2} / 0$. Note that $\mathrm{d} \tau^{2}$ is invariant under the Lorentz transformation (11). Then pairs of spacetime events could be classified into either time-like, $\mathrm{d} \tau^{2}>0$, or space-like, $\mathrm{d} \tau^{2} \leq 0$, with the time ordering of spacelike events not being uniquely defined. However this outcome is merely an artifact of the mixed space-time coordinates: $\mathrm{dT}$ is not the actual time interval.

\section{CONCLUSION}

Physics failed to discover the existence of a dynamical 3-space until very recently. This discovery changes all of physics. The dynamics has been revealed and extensive direct and indirect evidence, from laboratory $\mathrm{G}$ measuring experiments, to the expansion of the universe and without "dark matter" nor "dark energy", is now explained. The nature of the theory suggests that space is a quantum phenomenon and the occurrence of the fine structure constant in both quantum matter and space phenomena suggest that a grand unification of until now disparate phenomena, is emerging. As well the experimental data shows that it is a neo-Lorentzian relativity that explains relativistic effects, as absolute motion effects and that Newtonian gravity and its successor, General Relativity, fail as theories of gravity and, for GR, a theory of the universe. Of particular significance is that the Special Relativity formalism is exactly derivable from Galilean Relativity by a mere change of space and time coordinates. As well new quantum detectors for 3-space have been recently discovered, which are very simple and cheap. These have revealed, as have numerous earlier experiments, the existence of 3-space turbulence/waves, also known as "gravitational waves". These waves have significant amplitude and the larger fluctuations are seen to correlate with solar flares, earth-quakes and other novel phenomena. A major discovery is that the quantum to classical transition is induced by 3 -space fluctuations and is not a random process internal to a quantum system, as assumed in the usual interpretation of quantum theory. The simplest detectors for gravitational waves are zener diodes, where electrons tunnelling through a $\sim 10 \mathrm{~nm}$ barrier are split into two components: Subsequent localization of the wave function to one side or the other of the barrier is caused by 3 -space fluctuations.

\section{ACKNOWLEDGEMENT}

This report is from the Flinders University Gravitational Wave Detector Project, funded by an Australian Research Council Discovery Grant: Development and Study of a New Theory of Gravity.

\section{REFERENCES}

Cahill, R.T. and D. Kerrigan, 2011. Dynamical space: Supermassive galactic black holes and cosmic filaments. Progress Phys., 4: 79-82.

Cahill, R.T. and D. Rothall, 2012. Discovery of uniformly expanding universe. Progress Phys., 1: 63-68.

Cahill, R.T. and K. Kitto, 2003. Michelson-morley experiments revisited. Apeiron, 10: 104-117.

Cahill, R.T. and S. Deane, 2013. Dynamical 3-space gravitational waves: Reverberation effects. Progress Phys., 2: 9-11.

Cahill, R.T., 2005. Process Physics: From Information Theory to Quantum Space and Matter. 1st Edn., Nova Science Publishers, New York, ISBN-10: 1594543003, pp: 215.

Cahill, R.T., 2006. 3-Space inflow theory of gravity: Boreholes, black holes and the fine structure constant. Progress Phys., 2: 9-16.

Cahill, R.T., 2008. Unravelling lorentz covariance and the spacetime formalism. Progress Phys., 4: 1924.

Cahill, R.T., 2009a. Dynamical 3-Space: A Review. In: Ether Space-Time And Cosmology: New Insights into a Key Physical Medium, Levy, J. and M.C. Duffy (Eds.), Apeiron, Montreal, ISBN-10: 0973291184, pp: 135-200.

Cahill, R.T., 2009b. Combining NASA/JPL One-way optical-fiber light speed data with spacecraft earthflyby doppler-shift data to characterise 3-space flow. Progress Phys., 4: 50-64.

Cahill, R.T., 2011a. Dynamical 3-Space: Emergent Gravity. In: Should the Laws of Gravity be Reconsidered, Munera, H.A. (Ed.), Apeiron, Montreal, ISBN-10: 978-0-9864926-5-5, pp: 363376. 
Cahill, R.T., 2011b. Dynamical 3-Space: Cosmic filaments, sheets and voids. Progress Phys., 2: 4451

Cahill, R.T., 2012. Characterisation of low frequency gravitational waves from dual RF coaxial-cable detector: Fractal Textured dynamical 3-space. Progress Phys., 3: 3-10.

Cahill, R.T., 2013a. Dynamical 3-Space: Neo-lorentz relativity. Phys. Int., 4: 60-72. DOI: 10.3844/pisp.2013.60.72
Cahill, R.T., 2013b. Nanotechnology quantum detectors for gravitational waves: Adelaide to london correlations observed. Progress Phys., 4: 57-62.

Hertz, H., 1890. On the fundamental equations of electro-magnetics for bodies in motion. Wiedemann's Ann.

Rothall D.P. and R.T. Cahill, 2013. Dynamical 3-Space: Black holes in an expanding universe. Progress Phys., 4: 25-31. 